\title{
OTIOTOMTCS
}

Revista de economía, empresa y sociedad

EL IMPACTO DE AMAZON

\section{La logística como fuente de valor añadido al eCommerce}

\section{Xavier Budet Jofra}

Profesor colaborador del máster de Dirección de operaciones y logística integral (UOC)

\section{Alexis Pérez Gómez}

Profesor colaborador del máster de Dirección de operaciones y logística integral (UOC)

RESUMEN Las plataformas de eCommerce se convierten en actores cada vez más relevantes de la economía global, modificando los hábitos de compra y consumo, y provocando un impacto decisivo en el mercado de la distribución, fabricación y prestación de servicios logísticos.

La consolidación y mejora del eCommerce se basa en garantizar la excelencia en la experiencia de usuario, desde el proceso de compra hasta la entrega final del producto o del servicio solicitado. En este sentido, la logística es un factor clave.

La penetración de Amazon en los hogares ha sacudido fuertemente el escenario competitivo. Ha obligado a los grandes distribuidores (y fabricantes) a dar una respuesta que satisfaga las nuevas expectativas de servicio de los compradores, iniciando una exigente carrera para dominar la cadena de valor y la experiencia del cliente.

Con el presente artículo, los autores analizan el panorama actual, las repercusiones en la cadena de suministro y las tendencias que presentan las plataformas de eCommerce, identificando áreas de oportunidad generadas por las expectativas de clientes cada vez más exigentes. 
PALABRAS Clave eCommerce; logística; cadena de suministro; omnicanalidad; experiencia de compra; última milla

\section{Logistics as a source of added value in eCommerce}

ABSTRACT eCommerce platforms are becoming increasing significant players in the global economy, modifying shopping and consumption habits, and having a decisive impact on the market in terms of distribution, manufacture and the provision of logistics services.

The consolidation and improvement of eCommerce is underpinned by guaranteeing excellence throughout the user experience, from the shopping process right through to the final delivery of the product or service requested. In this respect, logistics is a key factor.

Amazon's penetration in households has strongly shaken up the scene in terms of the competition. It has forced large retailers (and manufacturers) to provide a response that fulfils shoppers' new service expectations, triggering a gruelling race to take control of the value chain and the customer experience.

In this article, the authors analyse the current panorama, the repercussions for the supply chain and the trends emerging in terms of eCommerce platform, identifying areas of opportunity generated by increasingly demanding customer expectations.

KEYWORDS eCommerce; logistics; supply chain; omni-channel; shopping experience; last mile

\section{Introducción}

El eCommerce, por definición, responde a las transacciones comerciales que tienen lugar en internet mediante páginas web y aplicaciones móviles (app) entre proveedores, vendedores, centros comerciales y clientes.

Desde sus orígenes en 1995, el eCommerce ha experimentado un continuo y acelerado crecimiento en los sectores retail, viajes y multimedia. Ha contado con la complicidad del adelanto tecnológico, las telecomunicaciones, los dispositivos móviles y las plataformas de venta en línea, experimentando una transición de entornos web a aplicaciones de uso en dispositivos móviles. El progreso tecnológico ha permitido la virtualización de conceptos físicos como por ejemplo los centros comerciales, las listas de la compra y la atención al cliente. Estos entornos virtuales 
permiten la compra descentralizada tanto de servicios como de productos entregados por medios electrónicos y/o servicios logísticos.

A pesar de que se prevé una cierta desaceleración en el ritmo de crecimiento de ventas eCommerce, este todavía presenta tendencias claramente alcistas en ciertos países de entre el 1,45 \% y el 39 \% según datos de la Global Ecommerce Foundation.

La cotización en el mercado de valores NASDAQ de la acción de Amazon es un fiel reflejo de la evolución del eCommerce: ha pasado de ser una promesa con muchos interrogantes y escepticismo a una fenomenal realidad con crecimiento exponencial (https://www.nasdaq.com/symbol/amzn/interactive-chart).

El eCommerce está revolucionando, pues, los hábitos de consumo, la experiencia de compra, la distribución y la venta de bienes y servicios, lo que ha provocado cambios significativos en la cadena de suministro.

Las empresas basadas en el canal en línea, los pure players, han generado unas expectativas y exigencias que han llevado la logística a un nuevo estadio. Esto está obligando a rediseñar procesos, activos, soluciones tecnológicas, organizaciones y redes de distribución. Como respuesta a este nuevo escenario competitivo, centrado en gran medida en la experiencia del cliente, los distribuidores han optado por una estrategia basada en facilitar al consumidor cualquier interacción: desde la comunicación hasta el proceso de compra (pasando por la búsqueda de información, las devoluciones, etc.). Esto ha conducido a un nuevo concepto: la omnicanalidad, sobre el que se profundizará a lo largo del presente artículo.

\section{Amazon abre la caja de los truenos}

Amazon nació con la voluntad de ser la librería del planeta, con la obsesión de conocer los hábitos de los consumidores y sobresalir en la experiencia de compra de los usuarios para poder sobrevivir. Muy pronto se dio cuenta de que, además de libros, podía vender cualquier otra cosa. Una vez llevaba un libro e iba conociendo el cliente, ¿por qué no aprovechar todas las sinergias y ofrecerle más cosas?

Y así es como Amazon empezó a introducir categorías y más categorías en su surtido, con un objetivo claro a largo plazo. Tomaba decisiones que no parecían lógicas, pero que hoy en día le han supuesto una ventaja competitiva: Amazon comprendió que sin el control de la cadena de suministro no podía sobresalir en experiencia del cliente.

Primero, creó una densa red de centros de distribución para conseguir ofrecer su surtido (concepto long tail) acortando plazos de entrega. Después, adquirió la 
compañía Kiva para automatizar estos centros, mejorando la calidad de pedidos al reducir costes operativos; y desarrolló algoritmos de predicción de demanda y pricing. Posteriormente, reorganizó la cadena de suministro y exploró nuevas formas de entrega para poder servir el mismo día (same-day-delivery) como servicio premium. En los últimos años, ha ido dando nuevos pasos hacia la innovación: probando drones, desarrollando aparatos para facilitar la confección de pedidos, reforzando la red de forwarding internacional (para controlar toda la cadena de suministro) y, finalmente, creando poco a poco una flota propia para complementar a los operadores tradicionales.

Amazon gana dinero desde el último cuatrimestre del año 2015 y su crecimiento exponencial amenaza, y asusta, a los actores de muchos sectores; especialmente, de la gran distribución de todo el mundo. Se puede afirmar que, dentro de la cadena de suministro, Amazon se ha convertido en la referencia.

\section{La respuesta de la gran distribución: la omnicanalidad}

A día de hoy, el efecto Amazon ya ha tenido efectos devastadores para muchos distribuidores. En los últimos años, en los Estados Unidos y en Europa, múltiples cadenas de tiendas han tenido que cerrar debido al cambio acelerado de hábitos de compra. En este último período, mientras las ventas del «canal tienda» se han estancado o menguado, las ventas del «canal online» están creciendo a dos dígitos.

A raíz de esto, la gran distribución (como por ejemplo Walmart, Tesco, Carrefour, etc.) se ha dado cuenta de que su extensa red de tiendas les podría dar una ventaja competitiva sobre Amazon, y el resto de operadores en línea, con la tienda jugando otros roles. ¿Se pueden generar sinergias entre los diferentes canales? Por un lado, la tienda podría ofrecer servicio y capilaridad y, por el otro, el canal online permitiría inmediatez y comodidad. La respuesta, por lo tanto, parece obvia, pero su implementación no es tan sencilla.

La omnicanalidad no es la oferta de productos empleando diferentes canales. De hecho, esto ya lo estaban haciendo estas empresas. La clave tiene que ser la experiencia de cliente integrada y consistente, sin importar desde qué canal quieren acceder. La realidad es que, por ejemplo, un cliente puede informarse en internet, ir a ver el producto a la tienda, llamar al servicio de atención al cliente por teléfono, hacer la compra virtualmente para recogerla en coche, etc. 
Figura 1. Concepto omnicanalidad

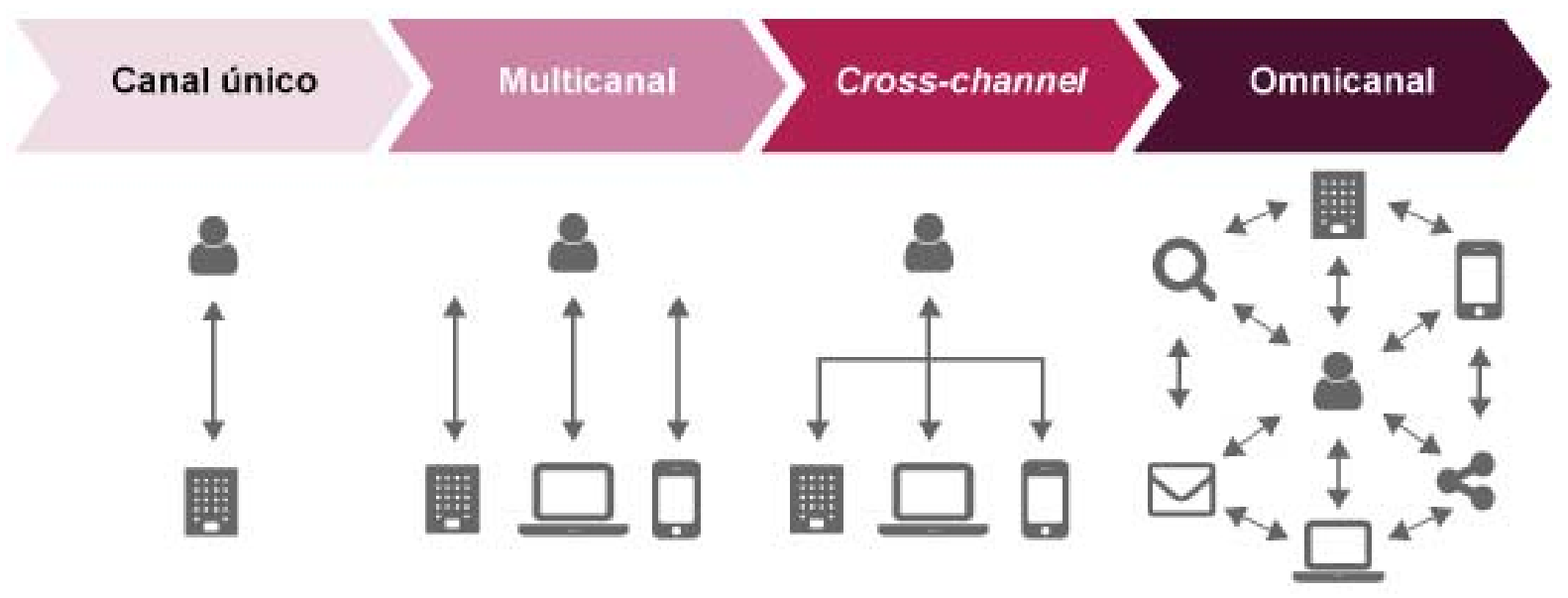

Fuente: http://loem.ca/en/omni-channel-strategy-new-eldorado-commerce/.

Aspectos clave y tendencias de la omnicanalidad

En un mundo donde los cambios son cada vez más acelerados, y los plazos más cortos, la omnicanalidad requiere velocidad y flexibilidad en los diferentes procesos y, por lo tanto, visibilidad y conectividad. Al mismo tiempo, sin embargo, esta necesidad de velocidad y flexibilidad pone en una gran exigencia a la cadena de suministro. Garantizar la eficiencia operacional por ser rentable se ha convertido en un reto mayúsculo.

A continuación, se desgrana este reto en los principales aspectos a considerar:

\section{Gestión de compras y de aprovisionamiento}

La relación con los proveedores tiene que pasar de ser puramente transaccional a una más colaborativa. Los proveedores pueden llegar a ser clave para mejorar el servicio al cliente, pero necesitan visibilidad y datos. ¿Cómo se pueden garantizar plazos de entrega suficientemente cortos? ¿Cómo se puede reducir el inventario en toda la cadena? ¿Tiene sentido plantear envíos directos desde los proveedores?

\section{Estrategia de posicionamiento y gestión del inventario en toda la red (al- macenes, centros de distribución o tiendas)}

Durante años, las empresas distribuidoras se han concentrado en optimizar el inventario en los diferentes eslabones de la cadena de suministro, que tenía un flujo muy lineal: de proveedor a centro de distribución, de centro de distribución a tienda, de tienda a cliente final. Los ciclos cada vez más cortos requieren enviar directamente al cliente desde cualquier punto. Los nuevos servicios de la tienda (preparación y envío, recogida, etc.) tienen también un impacto muy relevante en 
el inventario, pues la demanda de un canal puede ser muy diferente a la demanda en otro -tendencia, estacionalidad, frecuencia de compra, etc.- y la capacidad del distribuidor de dar respuesta a esta demanda desde cualquier punto de la red es capital. ¿Desde dónde «servirá» mejor el inventario? ¿Qué cantidad hay que asignar en cada tienda y en los centros de distribución para contener el capital circulante? ¿Cómo se pueden hacer las devoluciones? ¿Dónde y cómo se hará la comprobación, el repacking y se posicionará el producto devuelto? ¿Qué nivel de servicio se puede ofrecer en cada nodo de la red? ¿Cómo se consigue que haya sinergias, en vez de empeoramiento del servicio, de los dos canales que comparten stock? Todos estos elementos determinarán el siempre difícil equilibrio entre servicio y minimización del capital circulante.

\section{Preparación y envío de pedidos (fulfillment)}

La exigencia de reducir cada vez más los plazos de entrega ha provocado un replanteamiento de dónde hay que preparar los pedidos. ¿En un centro de distribución (aprovechando las sinergias de recursos tecnológicos y humanos, la menor cantidad de rupturas de stock, la fiabilidad y la eficiencia de la preparación) o en una tienda (aprovechando la proximidad al cliente, reduciendo costes de transporte y permitiendo una expansión a nuevas zonas más rápidamente)? Si se hace en tienda, ¿mejor desde una tienda abierta al público o en un centro cerrado (dark store)? Hay mucha variedad de modelos, incluso mixtos (por ejemplo, se puede empezar haciendo la preparación en la tienda, hasta que hay suficiente masa crítica para cambiar a un centro de distribución).

Por otro lado, el proceso que determina el servicio percibido, y supone la mayor parte del coste, es la entrega al cliente, lo que se conoce como la «última milla». En esta fase, además de escoger los vehículos más idóneos, hay que buscar el equilibrio entre el servicio deseado por el cliente (una hora exacta) y la eficiencia del repartidor. Estudios realizados en las ciudades de Londres y Helsinki han demostrado que pasar de franjas de 3 a 6 horas reduce los costes de transporte entre el 17 y el $24 \%$, o hasta el $50 \%$ si se prescinde totalmente de las franjas. Pero ¿es aceptable este escenario por parte del cliente? ¿Qué coste tiene repetir la entrega del pedido por ausencia del cliente? Depende, está claro, del índice de ausencias, y este puede llegar al 30 \%. Es por este motivo que han empezado a proliferar otros sistemas de entrega en puntos fijos (puntos de recogida, comercios adscritos a una red, taquillas electrónicas), que pueden reducir el coste de distribución hasta el $60 \%$.

\section{Gestión de la información}

Hoy en día, la información fiable a tiempo real es clave para poder optimizar las operaciones y minimizar el stock distribuido. ¿Qué soluciones adicionales reque- 
rirán los diferentes procesos de la cadena de suministro? ¿Qué tecnologías nos pueden ayudar a hacer análisis cuidadosos (predictivos, con escenarios what-if) como apoyo a la toma de decisiones?

Es obvio que el eCommerce ha cambiado la logística completamente. De una logística centralizada, con miles de referencias y servicio en palés transportados por camiones, se ha pasado a una logística distribuida, con millones de referencias y servicio en cajas entregadas con furgonetas (o, incluso, motocicletas y bicicletas).

En este entorno es tan importante el servicio como las características del producto: el posicionamiento dentro de la cadena de suministro y la entrega pasan a ser clave para garantizar la disponibilidad de lo que quiere el cliente en el momento que lo quiere... y a un precio competitivo.

En medio de este escenario turbulento de elevada competitividad y exigencia, se ha iniciado una carrera por la omnicanalidad. Por un lado, nuevos actores están entrando en acción, como las empresas especializadas en comprar en diferentes establecimientos (generalmente de alimentación) y entregar en pocas horas al cliente. Las más conocidas son las americanas Instacart o Shipt; en nuestro país tenemos también casos como Deliberry. Esta capacidad de capilaridad y rapidez en la entrega son clave hoy en día y, por lo tanto, la gran distribución ha buscado acuerdos de partenariado con estas empresas (o directamente las han comprado, como la reciente compra de Shipt por parte de la empresa Target) para poder competir con Amazon y otras pure players.

Por otro lado, la gran distribución compra pure players (por ejemplo, el distribuidor más grande del mundo, Walmart, ha comprado Jet y Bonobos en el último año), los fabricantes también quieren introducirse en la venta directa (por ejemplo, Unilever compra Dollars Shave Club) y compañías tecnológicas como Google se alían con distribuidores (con Walmart, hace pocos meses) para desarrollar el Google's Shopping Express y desafiar a Amazon en la compra en línea.

Finalmente, los grandes pure players globales, como Amazon, Jd.com y Alibaba, han empezado a comprar empresas distribuidoras para contrarrestar las iniciativas de la gran distribución y ser también omnicanal. El caso más conocido, por ejemplo, ha sido la reciente compra de Whole Foods por parte de Amazon.

En el actual entorno competitivo, conseguir una experiencia omnicanal consistente ha representado un diferencial y, por lo tanto, ha pasado a ser crucial desarrollar una cadena de suministro centrada en la experiencia del cliente. 


\section{Calidad de servicio como valor añadido a la cadena de aprovisionamiento}

Se entiende como calidad de servicio el cumplimiento de todos los parámetros que tienen que garantizar la experiencia del cliente durante el proceso de compra; desde la navegación web, la información facilitada, el apoyo al cliente ofrecido preventa/posventa, hasta la logística para alcanzarlo con el producto comprado.

Para lograr la mejora continua y la excelencia en la calidad de servicio, la logística juega un papel fundamental. Además, optimizar las operaciones logísticas permite reducir los costes repercutidos sobre el precio de venta asumido por el cliente, o bien soportados por el vendedor con descuentos por volumen con los distribuidores subcontratados.

Uno de los elementos de máxima atención y que conjuga ambas, la ineficiencia operacional y también la satisfacción del cliente, es la reducción de los problemas que suceden en la «última milla».

Según UPS (United Parcel Service), el 50 \% de las entregas a domicilio están relacionadas con entregas de última milla del sector eCommerce, viajando de centros de distribución o tiendas pequeñas hasta el punto de demanda o cliente. En el año 2014 este envío se lograba en ocho días de media; en la actualidad son cinco. Las expectativas de entrega inmediata fuerzan a las empresas a incrementar su riesgo en inventario, teniendo que complementar este servicio con devoluciones gratuitas.

La entrega de la última milla es un gran negocio. Solo en el año 2016, las empresas de eCommerce se gastaron en torno a 65.000 millones de dólares para preparar, empaquetar y transportar productos, logrando una facturación en línea de unos 370.000 millones. Estos costes logísticos son significativamente superiores si se comparan con los típicos de la tienda tradicional o los grandes almacenes (media del $17,5 \%$ y $6,5 \%$, respectivamente).

Además de la presión en costes para mejorar la competitividad, de la exigencia de los plazos y de la puntualidad de entrega, además de la ausencia de errores en la preparación, hay que introducir un nuevo elemento: los picos de demanda provocados por la política comercial (Black Friday, Cyber Monday, Navidad, etc.). Estos picos obligan a las empresas que realizan las operaciones de la última milla (y las que las contratan) a buscar nuevas fórmulas de entrega como el crowdsourcing (Uber es un buen ejemplo). 
Figura 2. Evolución del Black Friday en España

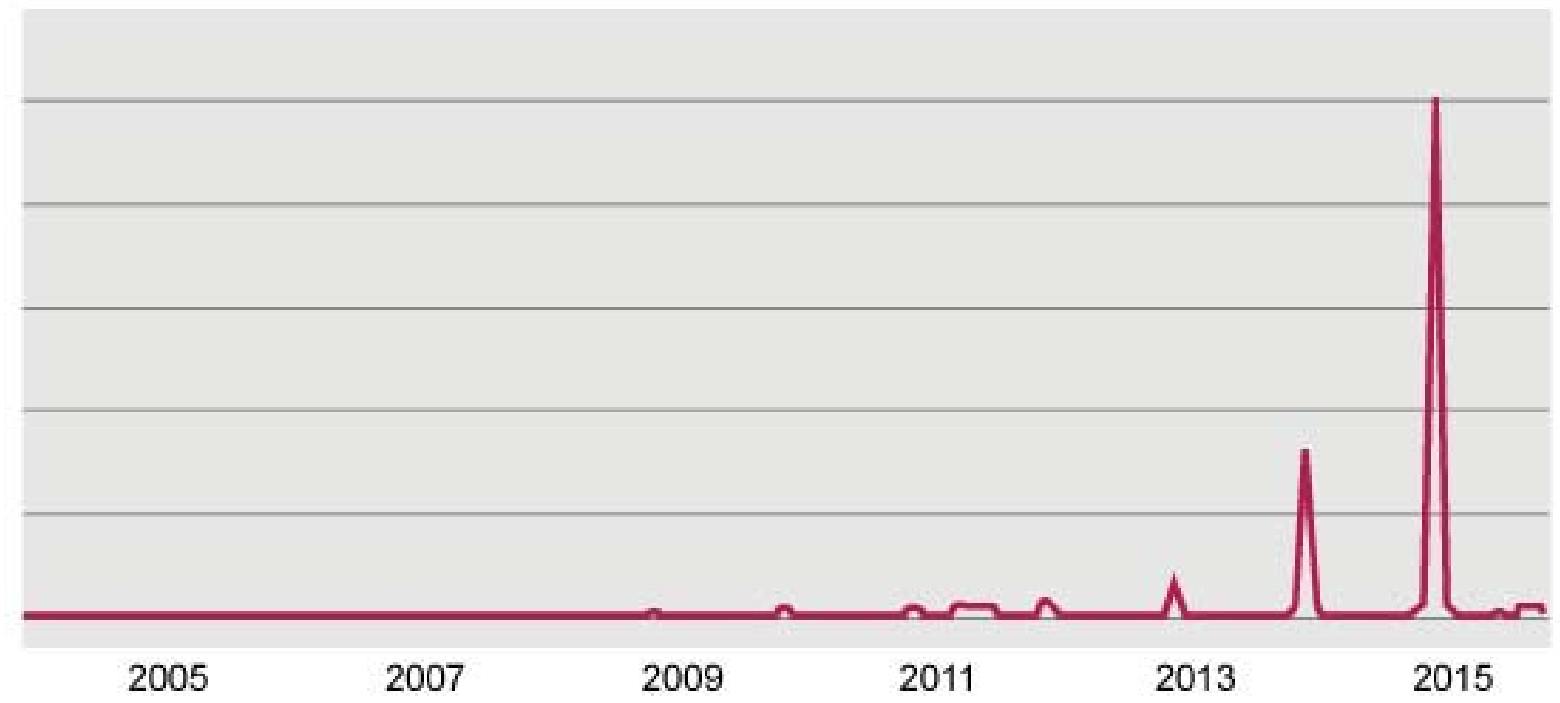

Fuente: Wikimedia Commons.

En la anterior figura se observa cómo las ventas durante campañas como la del Black Friday han aumentado espectacularmente en los últimos años solo en España. Reflejan esta variabilidad de demanda más otros factores como los antes descritos, donde los envíos a temperatura controlada (alimentación, farmacia, etc.), la congestión de las grandes ciudades y la reticencia de una gran mayoría de clientes a pagar la entrega (que tienen que ser absorbidos por el margen de los distribuidores y/o de los operadores logísticos) aumentan la complejidad de las operaciones de la última milla y ponen presión en los costes de distribución.

Todos estos requerimientos están creando fuertes tensiones entre los distribuidores, que buscan eficiencia, disponibilidad, rapidez y visibilidad, y los operadores logísticos, que necesitan volúmenes consistentes, rentabilidad de sus activos y una cierta predictibilidad.

Por otro lado, los clientes, especialmente millennials y urbanitas, piden finalizar sus compras en línea con entregas rápidas de productos de rápido consumo como comida o consumibles, pero también en el sector del lujo. Un 60 \% de los millennials espera que el tipo de entrega en un mismo día sea una opción a elegir en su compra, y más de un 60 \% está dispuesto a pagar más por este servicio.

Las tendencias en el eCommerce indican que las entregas del mismo día serán cada vez más demandadas, y se convertirán en la actividad propia de repartidores locales, que absorberán esta parte del negocio de los grandes proveedores logísticos internacionales. Aun así, es probable que sean los propios gigantes logísticos quienes subcontraten esta infraestructura local. Se espera que UPS, FedEx o Amazon inviertan en startups de reparto local y en la evolución de tecnologías que lo permitan (Budet y Pérez, 2018). 


\section{Conclusiones}

El eCommerce y las nuevas tecnologías están revolucionando la forma de vender y comprar productos. Los cambios, cada vez más acelerados, han impactado de una forma decisiva en la cadena de suministro, trayéndola a un nuevo estadio.

La competitividad creciente entre los especialistas de eCommerce, pure players y los amenazados «viejos» gigantes de la distribución está llevando los procesos logísticos a elevadas exigencias. La irrupción de la omnicanalidad, por ejemplo, surge para dar respuesta a las necesidades y mejorar la experiencia del cliente desde cualquier canal.

En este entorno, quien no domine la cadena de suministro quedará fuera de juego. Esto supone tener que implantar la tecnología adecuada, saberla integrar, atraer personas con habilidades para extraer el máximo provecho, gestionar con eficacia la información, establecer relaciones de partenariado con otros actores clave y tener el dominio de los procesos críticos de la cadena de suministro.

\section{Datos de interés}

- Se prevé que en el año 2025 haya una media de entre 50 y 60 millones de paquetes entregados al día a consumidor final (B2C), solo en los Estados Unidos (McKinsey).

- Con respecto al crecimiento de la demanda de entregas instantáneas o en el mismo día, el 70 \% de los consumidores prefieren la opción más barata (McKinsey).

- En el año 2025, el 80 \% de los paquetes B2C serán entregados por vehículos autónomos, incluyendo drones que sirvan en zonas rurales (McKinsey).

- El $72,1 \%$ de los clientes han tenido uno o más incumplimientos de los plazos de entrega (Trusted Shops).

- IDC Manufacturing Insights prevé, a finales del año 2020, que el $50 \%$ de todas las cadenas de suministro tengan la capacidad de habilitar envíos directos a clientes finales y/o entrega a domicilio.

- La tasa de entrega fallida se sitúa en España entre el $20 \%$ y el 30 \%, según el Libro Blanco de la Logística de Adigital.

- Según una encuesta realizada en el año 2017 a doscientos directivos de la cadena de suministro de empresas de distribución americanas, el 83 \% consideraba que la experiencia de cliente tenía que ser uno de los ejes principales de su estrategia. No obstante, un $66 \%$ consideraba que no tenía las soluciones tecnológicas adecuadas para hacer frente a este reto, y un $79 \%$ decía que la eficiencia era todavía el principal objetivo o uno de los principales (ETF-Convey). 
- Penetración del canal online a enero de 2018 (porcentaje de ventas en línea): Reino Unido, 17 \%; Estados Unidos, 15 \%; Alemania, 14 \%; España, 4 \% (La Vanguardia).

- En 2017, Amazon, con casi 200.000 millones de dólares, ha capturado un $44 \%$ de las ventas de comercio electrónico en los Estados Unidos; esto supone el $4 \%$ del total de ventas de retail (One Click Retail).

- Comparación de Amazon frente a Walmart:

\begin{tabular}{|l|l|l|}
\hline & \multicolumn{1}{|c|}{ Amazon } & \multicolumn{1}{c|}{ Walmart } \\
\hline Año fundación & 1994 & 1962 \\
\hline Trabajadores & 550.000 & 2.300 .000 \\
\hline Ventas en 2017 (M\$) & 161.000 & 485.000 \\
\hline Promedio crecimiento 5 años & $21 \%$ & $1 \%$ \\
\hline Referencias (millones) & 320 & 50 \\
\hline Tiendas & 400 (3 países) & 11.000 (28 países) \\
\hline
\end{tabular}

Un $12 \%$ de las ventas de Walmart son por el canal online.

Un $10 \%$ de las ventas de Amazon son por el canal tienda (Whole Foods, básicamente).

\section{Referencias bibliográficas}

BUDET J. X.; PÉrez, A. (2018). «Innovacions tecnològiques en la cadena de subministrament aplicades a l'eCommerce». Oikonomics. N. ${ }^{\circ} 9$.

ECOMMERCE FOUNDATION (2017). Global Ecommerce Report 2017. <www. ecommercefoundation.org/reports>.

FERNIE, J.; SPARKS, L.; McKINNON A. C. (2010). «Retail logistics in the UK: past, present and future». International Journal of Retail \& Distribution Management. Vol. 38, núms. 11-12.

HU, M.; MONAHAN, S. (2016). «US e-Commerce Trends and the Impact on Logistics". A.T. Kearney, [artículo en línea]. <https://www.atkearney.com/retail/ article?/a/us-e-commerce-trends-and-the-impact-on-logistics>

HALLOWELL, R.; (2002). "Virtuous Cycles: Improving Service and Lowering Costs in E-commerce». Harvard Business School. Module Teaching Note 802-169.

PRASHAR, A. (2017). «Shopclues: Turning logistics into a competitive advantage». MDI Management Development Institute, Gurgaon and Richard Ivey School of Business Foundation. W17389. 
PUNAKIVI, M.; YRJÖLÄ, H.; HOLMSTRÖM, J. (2001). «Solving the last mile issue: reception box or delivery box?» International Journal of Physical Distribution \& Logistics Management. Vol. 31, n. ${ }^{\circ} 6$.

ZHU, F.; LANE, D. (2016). «SF Express: From Delivery to E-Commerce». Harvard Business School Case 616-003.

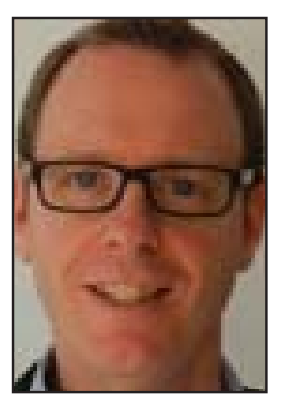

\section{Xavier Budet Jofra xbudet@uoc.edu Profesor colaborador del máster de Dirección de operaciones y logística integral (UOC)}

Ingeniero industrial superior por la Escuela Técnica Superior de Ingenieros Industriales de Barcelona (UPC), MBA por ESADE (Barcelona) y CSCP por Apics (Estados Unidos). Actualmente es director de Logística - Canal Online en La Sirena Alimentación Congelada S.L.U. También es profesor colaborador, tutor de proyectos en estudios de grado y máster relacionados con la cadena de suministro y autor de publicaciones de la UOC.

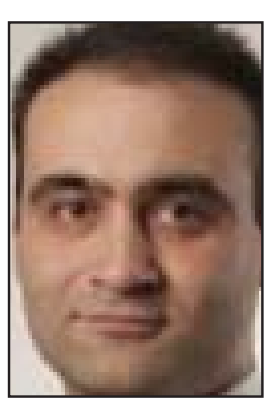

\section{Alexis Pérez Gómez} aperezgomez3@uoc.edu Profesor colaborador del máster de Dirección de operaciones y logística integral (UOC)

MBA por ESADE (Barcelona y McCombs Austin, Texas). Ingeniero industrial superior por la Escuela Politécnica Superior de la Universidad de Gerona. Ingeniero técnico en Informática de Sistemas por la Escuela Politécnica Superior de la Universidad de Gerona. Actualmente, es responsable de producción, abastecimiento de material y calidad de la actividad de reparaciones y apoyo al cliente de Europa, Medio Oriente y África en HP Inc. También es profesor colaborador, tutor de proyectos en estudios de grado y master relacionados con cadena de suministro y autor de publicaciones de la UOC. 
Los textos publicados en esta revista están sujetas -salvo que se indique el contrario- a una licencia de Reconocimiento 3.0 España de Creative Commons. Podéis copiarlos, distribuirlos, comunicarlos públicamente y hacer obras derivadas siempre que reconozcáis los créditos de las obras (autoría, nombre de la revista, institución editora) de la manera especificada por los autores o por la revista. La licencia completa se puede consultar en http://creativecommons.org/licenses/by/3.0/es/deed.ca.

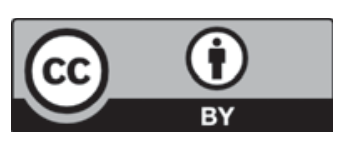

